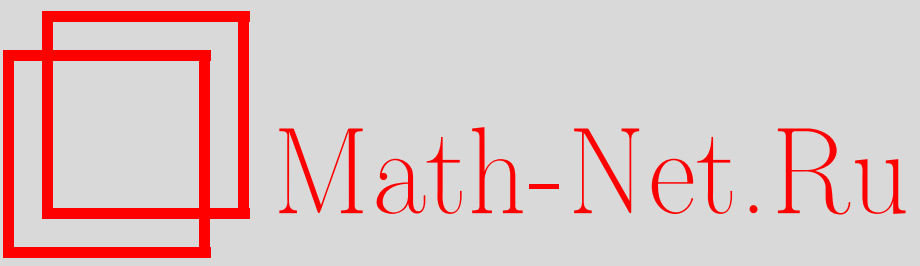

А. М. Семихатов, Б. Л. Фейгин, Резольвенты унитарных представлений $N=2$ алгебры Вирасоро в терминах свободных полей. II. Резольвента-бабочка, ТM $\Phi$, 1999, том 121, номер 2, 244-257

DOI: https://doi.org/10.4213/tmf805

Использование Общероссийского математического портала Math-Net.Ru подразумевает, что вы прочитали и согласны с пользовательским соглашением

http://www.mathnet.ru/rus/agreement

Параметры загрузки:

IP : 3.85 .73 .92

26 апреля 2023 г., 17:41:31 


\title{
РЕЗОЛЬВЕНТЫ УНИТАРНЫХ ПРЕДСТАВЛЕНИЙ $N=2$ АЛГЕБРЫ ВИРАСОРО В ТЕРМИНАХ СВОБОДНЫХ ПОЛЕЙ. ІІ. РЕЗОЛЬВЕНТА-БАБОЧКА
}

\author{
Построена резольвента нового типа для унитарных представлений $N=2$ суперкон- \\ формной алгебры, реализованных в пространстве свободных полей с двумя фермион- \\ ными экранируюшими операторами.
}

\section{1. ВВЕДЕНИЕ}

В данной статье продолжается исследование [1] резольвент унитарных представлений $N=2$ суперконформной алгебры, построенных в модулях свободных полей; мы отсылаем читателя к работе [1] за мотивировками и дополнительными ссылками на литературу. Резольвенты представлений $N=2$ суперконформной алгебры, реализованных в пространствах свободных полей, позволяют выделять неприводимые представления как когомологии некоторого комплекса (на более физическом языке когомологии "БРСТ-оператора"). Различные реализации через свободные поля приводят при этом к различным резольвентам.

До сих пор известные из различных контекстов резольвенты неприводимых представлений через свободные поля имели "линейную" структуру, подобно резольвенте Фельдера [2] (так называемые двусторонние резольвенты [3-5]). Это относится и к резольвенте из [1], хотя она и не является фельдеровской. Наличие "бозонизации" [6-8] $N=2$ алгебры, в которой возникают два фермионных экранируюших оператора, позволяет предполагать некоторую другую структуру резольвенты, в которой стрелки различного типа отвечали бы двум различным экранируюшим операторам. Такая резольвента и строится в данной работе. Она имеет структуру, из-за которой мы называем ее "ба-

\footnotetext{
* Отделение теоретической физики им. И.Е. Тамма, Физический институт им. П. Н. Лебедева РАН, Москва, Россия

${ }^{\dagger}$ Институт теоретической физики им. Л. Д. Ландау РАН, Черноголовка, Россия
} 
бочкой" (butterfly resolution):

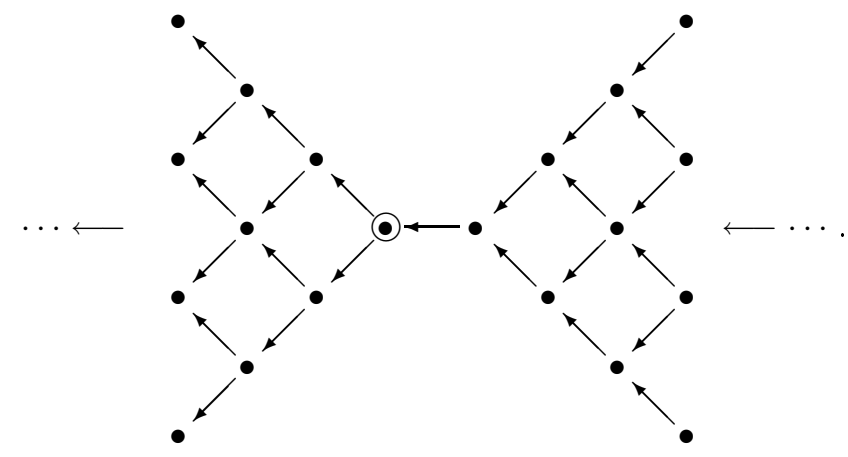

Задача о когомологиях для этой резольвенты также решается, и оказывается, что когомологии сосредоточены в единственном модуле (левом "глазу") и даются унитарным $N=2$ представлением (теорема 3.1 ниже). Путем "сминания крыльев" резольвента-бабочка переходит в двустороннюю резольвенту из работы [1] (а после складывания "крыльев" - в "массивную" резольвенту из [9], как объясняется в дальнейшем).

С более обшей точки зрения различные реализации в терминах свободных полей являются частными случаями трехбозонной реализации $N=2$ (или $\widehat{s \ell}(2))$ алгебры. Анализ резольвенты для общей трехбозонной реализации, который мы не ставим здесь своей целью, должен включать случаи, когда имеются:

1) бозонный и фермионный экранирующие операторы, образуюшие нильпотентную подалгебру квантовой группы $s \ell(2 \mid 1)_{q}$, и еше один бозонный экранирующий оператор, коммутируюший с первыми двумя;

2) два фермионных экранируюших оператора и еше один бозонный, не являюшийся вертексным оператором.

Что касается "s $\ell(2 \mid 1)_{q}$-пары" в случае 1 , то мы надеемся рассмотреть ее в будушем, в то время как коммутирующие друг с другом бозонный и фермионный экранируюшие операторы приводят в конце коншов к резольвенте типа фельдеровской, поскольку фермионный экранирующий оператор выделяет $\beta \gamma$-систему, после чего бозонный экранирующий оператор действует, как в работах $[3,4]$. Случай 2 не изучен в должной обшности, но рассматриваемая нами резольвента является важным частным случаем - случаем интегрируемых представлений, когда фермионные экранируюшие операторы коммутируют друг с другом.

Мы широко пользуемся обозначениями и соглашениями работы [1], ссылаясь на формулы оттуда как (I-s.n) и на разделы как I- $s$, где $s \in\{1,2,3,4,5, \Pi\}$ и $n \in \mathbb{N}$. Это позволяет нам сразу перейти в разделе 2 к интересуюшей нас реализации $N=2$ алгебры через свободные поля (которую мы называем симметричной, поскольку фермионные операторы $\mathcal{G}$ и $\mathcal{Q}$ выражаются через свободные поля почти симметричным образом). В разделе 3 описывается построение резольвенты, а в разделе 4 устанавливаются ее свойства (в частности, связь с резольвентой из [1]), позволяюшие найти ее когомоло- 
гии. Мы напомним только коммутационные соотношения $N=2$ алгебры:

$$
\begin{aligned}
{\left[\mathcal{L}_{m}, \mathcal{L}_{n}\right] } & =(m-n) \mathcal{L}_{m+n}, \quad\left[\mathcal{H}_{m}, \mathcal{H}_{n}\right]=\frac{\mathrm{c}}{3} m \delta_{m+n, 0}, \\
{\left[\mathcal{L}_{m}, \mathcal{G}_{n}\right] } & =(m-n) \mathcal{G}_{m+n}, \quad\left[\mathcal{H}_{m}, \mathcal{G}_{n}\right]=\mathcal{G}_{m+n}, \\
{\left[\mathcal{L}_{m}, \mathcal{Q}_{n}\right] } & =-n \mathcal{Q}_{m+n}, \quad\left[\mathcal{H}_{m}, \mathcal{Q}_{n}\right]=-\mathcal{Q}_{m+n}, \\
{\left[\mathcal{L}_{m}, \mathcal{H}_{n}\right] } & =-n \mathcal{H}_{m+n}+\frac{\mathrm{c}}{6}\left(m^{2}+m\right) \delta_{m+n, 0} \\
\left\{\mathcal{G}_{m}, \mathcal{Q}_{n}\right\} & =\mathcal{L}_{m+n}-n \mathcal{H}_{m+n}+\frac{\mathrm{c}}{6}\left(m^{2}+m\right) \delta_{m+n, 0}
\end{aligned}
$$

(где $m, n \in \mathbb{Z}, \mathcal{L}_{n}$ и $\mathcal{H}_{n}-$ бозоны, а $\mathcal{G}_{n}$ и $\mathcal{Q}_{n}$ - фермионы и с $=3(1-2 / p)$ - центральный заряд).

\section{2. “СИММЕТРИЧНАЯ" РЕАЛИЗАЦИЯ}

Рассмотрим реализацию $N=2$ алгебры из работ [6-8], которая принимает следующий вид в обозначениях, соответствующих (1.2):

$$
\begin{aligned}
\mathcal{G}(z) & =C(z) A(z)-\partial C(z), \\
\mathcal{Q}(z) & =B(z) \bar{A}(z)-\frac{1}{p} \partial B(z), \\
\mathcal{H}(z) & =\bar{A}(z)-\frac{1}{p} A(z)-B(z) C(z), \\
\mathcal{T}(z) & =\partial B(z) C(z)+\bar{A}(z) A(z)-\partial \bar{A}(z) .
\end{aligned}
$$

Здесь операторные произведения свободных полей заданы соотношениями

$$
\bar{A}(z) A(w)=\frac{1}{(z-w)^{2}}, \quad B(z) C(w)=\frac{1}{z-w}
$$

и обозначено $\partial=\partial / \partial z$. Разложение по модам имеет вид

$$
\begin{aligned}
A(z) & =\sum_{n \in \mathbb{Z}} A_{n} z^{-n-1}, & \bar{A}(z) & =\sum_{n \in \mathbb{Z}} \bar{A}_{n} z^{-n-1}, \\
B(z) & =\sum_{n \in \mathbb{Z}} B_{n} z^{-n}, & C(z) & =\sum_{n \in \mathbb{Z}} C_{n} z^{-n-1} .
\end{aligned}
$$

ЛЕмма 2.1. $N=2$ спектральный поток индуцируется следующими преобразованиями свободных полей, входящих в (2.1):

$$
\begin{aligned}
C_{n} & \mapsto C_{n+\theta}, \quad A_{n} \mapsto A_{n}+\theta \delta_{n, 0}, \\
B_{n} \mapsto B_{n-\theta}, & \bar{A}_{n} \mapsto \bar{A}_{n}-\frac{\theta}{p} \delta_{n, 0} .
\end{aligned}
$$


ДокАЗАТЕЛЬСТво получается непосредственно; единственным заслуживающим упоминания обстоятельством является то, что при указанных преобразованиях составные $B C$-операторы приобретают дополнительные вклады, происходяшие из нормального переупорядочения (которое опускается в обозначениях):

$$
(B C)_{n} \mapsto(B C)_{n}-\theta \delta_{n, 0}, \quad(\partial B C)_{n} \mapsto(\partial B C)_{n}-\theta(B C)_{n}+\frac{\theta^{2}+\theta}{2} \delta_{n, 0}
$$

Имеются два фермионных экранирующих оператора

$$
S_{B}=\frac{1}{2 \pi i} \oint B e^{X}, \quad S_{C}=\frac{1}{2 \pi i} \oint C e^{p \bar{X}},
$$

где $\partial X=A$ и $\partial \bar{X}=\bar{A}$. Очевидно, что экранируюшие операторы не меняются при преобразованиях (2.3)

Введем теперь модули над этими свободными полями. Пусть $\Lambda=\Lambda_{0}(0)$ обозначает $B C$-модуль, порожденный из $|0\rangle_{B C}$ (см. (I-3.9) и (I-3.11), где теперь $\lambda=0$ ). Пусть состояние $|\bar{a}, a\rangle_{\bar{A} A}$ таково, что

$$
\begin{gathered}
\bar{A}_{0}|\bar{a}, a\rangle_{\bar{A} A}=\bar{a}|\bar{a}, a\rangle_{\bar{A} A}, \quad A_{0}|\bar{a}, a\rangle_{\bar{A} A}=a|\bar{a}, a\rangle_{\bar{A} A}, \\
\bar{A}_{\geqslant 1}|\bar{a}, a\rangle_{\bar{A} A}=A_{\geqslant 1}|\bar{a}, a\rangle_{\bar{A} A}=0,
\end{gathered}
$$

и пусть $\mathfrak{H}_{\bar{a}, a}-$ фоковский модуль, порожденный из $|\bar{a}, a\rangle_{\bar{A} A}$. Определим пространство представления свободных полей

$$
\mathbb{G}_{r, p}=\Lambda \otimes \bigoplus_{m, n \in \mathbb{Z}} \mathfrak{H}_{n, m p+r-1} .
$$

Именно в нем мы выделим унитарное представление $\mathfrak{K}_{r, p ; 0}$. Другие представления $\mathfrak{K}_{r, p ; \theta}$ будут получаться применением спектрального потока в соответствии с (2.3).

Обозначения для $N=2$ модулей. Подобно принятым в п. I-3.3 соглашениям, $\widetilde{\mathfrak{U}}_{h, \ell, p ; \theta}(M, \bar{a}, a)$ обозначает модуль, порожденный из $|M\rangle_{B C} \otimes|\bar{a}, a\rangle_{\bar{A} A} \in \mathbb{G}_{r, p}$ в случае, ког да $|M\rangle_{B C} \otimes|\bar{a}, a\rangle_{\bar{A} A} \doteq|h, \ell, p ; \theta\rangle$ (знак $\doteq$ означает, что состояние $|M\rangle_{B C} \otimes|\bar{a}, a\rangle_{\bar{A} A}$ удовлетворяет твистованным массивным условиям старшего веса (I-П.10)-(I-П.12)), а $\widetilde{\mathfrak{V}}_{h, p ; \theta}(M, \bar{a}, a)$ обозначает такой модуль в случае, когда $|M\rangle_{B C} \otimes|\bar{a}, a\rangle_{\bar{A} A} \doteq|h, p ; \theta\rangle_{\text {top }}$. Мы иногда будем опускать аргументы $(M, \bar{a}, a)$, вместо этого явно указывая, из каких векторов порождается данный модуль. Заметим, что нам встретятся $N=2$ модули, которые не порождаются из одного вектора.

В пространстве $\mathbb{G}_{r, p}$ возьмем состояние $|0\rangle_{B C} \otimes|0, r-1\rangle_{\bar{A} A}$, которое удовлетворяет топологическим (нетвистованным) условиям старшего веса по отношению к $N=2$ генераторам (2.1):

$$
|0\rangle_{B C} \otimes|0, r-1\rangle_{\bar{A} A} \doteq\left|\frac{1-r}{p}, p\right\rangle_{\text {top }} .
$$




\section{3. РЕЗОЛЬВЕНТА-БАБОЧКА}

Пусть $\widetilde{\mathfrak{V}}_{\frac{1-r}{p}, p}(0,0, r-1)$ есть $N=2$ модуль, порожденный из $(2.9)$. Он является подмодулем в "центральном" члене $\odot$ (где и сконцентрированы когомологии) в резольвенme-бабочке $e^{1)}$ :

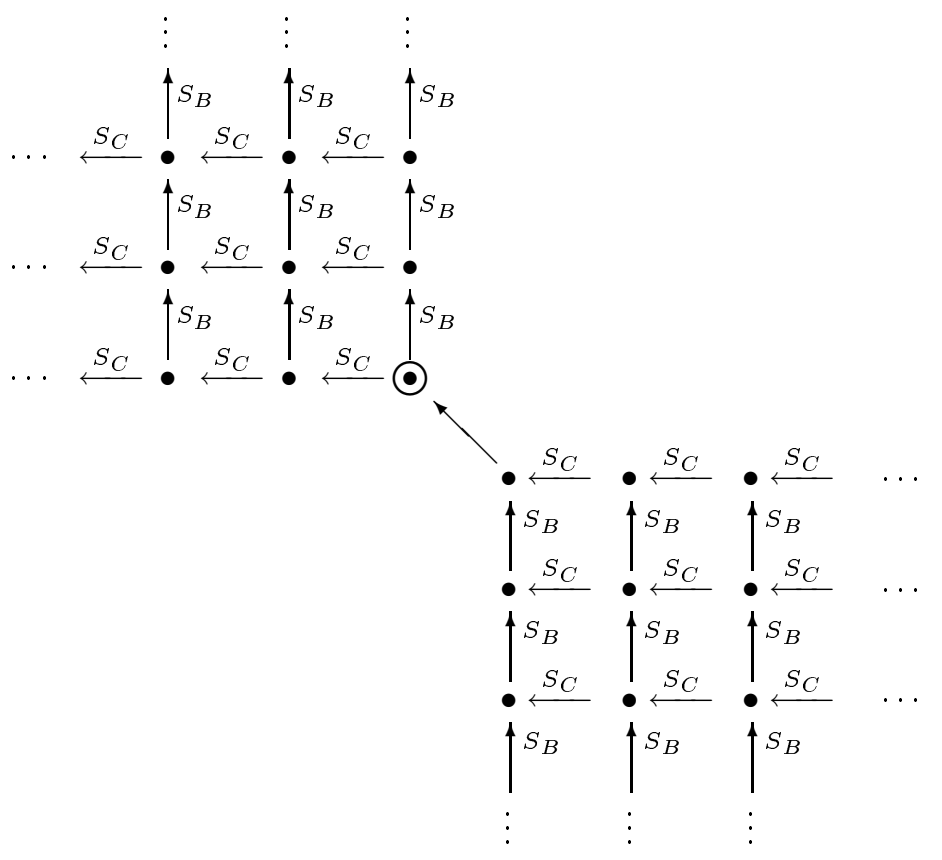

Модули на правом крыле имеют вид $\widetilde{\mathfrak{U}}_{\frac{r+1}{p}-n-m, 0, p ; n p-r}(n p-r,-m, r-n p-1)$ и нумеруются натуральными числами $m$ и $n$, где $n$ нумерует столбцы ( $n=1$ для самого левого столбца), а $m$ - строки ( $m=1$ для верхней строки). На левом крыле модули нумеруются неотрицательными целыми $m$ и $n$, причем $m=0$ соответствует нижней строке, а $n=0$ - правому столбцу. Каждый $(m, n)$-модуль есть результат склейки модулей, порожденных из состояний $|-r-n p\rangle_{B C} \otimes|m, n p+r-1\rangle_{\bar{A} A}$ и $|m p+1\rangle_{B C} \otimes$ $|m, n p+r-1\rangle_{\bar{A} A}$. В частности, $\odot$-модуль получен склейкой $N=2$ модулей, порожденных из векторов $|-r\rangle_{B C} \otimes|0, r-1\rangle_{\bar{A} A}$ и $|1\rangle_{B C} \otimes|0, r-1\rangle_{\bar{A} A}$, у которых имеется обший подмодуль, порожденный из вектора (2.9).

Теорема 3.1. Диаграмма (3.1), состоящая из $N=2$ представлений на $\mathbb{G}_{r, p}$, яв-

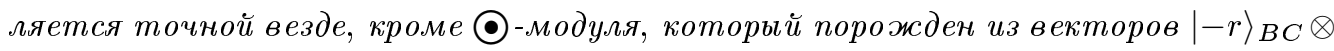
$|0, r-1\rangle_{\bar{A} A} u|1\rangle_{B C} \otimes|0, r-1\rangle_{\bar{A} A}$. Когомологии в әтом члене даются унитарныц $N=2$ представлением $\mathfrak{K}_{r, p}$.

\footnotetext{
1) Или, быть может (после поворота на $135^{\circ}$ ), в "песочных часах".
} 
ЗАМЕЧАНИЕ 3.2. Мы произвольным образом выбрали целое число $r$ из интервала $1 \leqslant r \leqslant p-1$ и определили зависяшее от $r$ пространство $\mathbb{G}_{r, p}$. Рассматривая вместо этого пространство

$$
\mathbb{G}_{*, p}=\Lambda \otimes \bigoplus_{m, n \in \mathbb{Z}} \mathfrak{H}_{n, m},
$$

получаем когомологии $\bigoplus_{r=1}^{p-1} \mathfrak{K}_{r, p}$.

ЗАМЕчАнИЕ 3.3. $N=2$ спектральньй поток действует на состояниях из $\mathbb{G}_{*, p}$ как

$$
|n\rangle_{B C} \otimes|\bar{a}, a\rangle_{\bar{A} A} \mapsto|n+\theta\rangle_{B C} \otimes\left|\bar{a}+\frac{\theta}{p}, a-\theta\right\rangle_{\bar{A} A} .
$$

Это позволяет применить спектральньй поток к данным из теоремы 3.1 (с подвергнутым соответствуюшему отображению пространством $\left.\mathbb{G}_{r, p}\right)$ с тем, чтобы получить все унитарные представления $\mathfrak{K}_{r, p ; \theta}$ из раздела I-2. Поэтому мы без потери обшности можем работать с выбранным значением твиста (и тем самым с указанным выше пространством $\left.\mathbb{G}_{r, p}\right)$, фиксированным нашим выбором состояния (2.9).

ДОКАЗАТЕЛЬСТВО ТЕОРЕМЫ 3.1. Конструкция резольвенты основана на заряженных особых векторах (I-П.14) и на топологических особых векторах (I-П.5) и (I-П.6) (соответствуюших значению $s=1$ ). В реализации свободных полей эти особые векторы могут обращаться в нуль. В этом случае оказывается возможным найти коособый вектор в той же градуировке. Если же особый вектор не равен нулю, то мы рассмотрим соответствуюший подмодуль и найдем уже в нем подобные особые векторы. Для состояния (2.9) и для других возникаюших в процессе построения состояний старшего веса мы находим условия аннигиляции, которым они удовлетворяют. Эти условия могут оказаться или твистованными топологическими условиями старшего веса (I-П.1), или же только твистованными массивными условиями старшего веса (І-П.11). Далее мы вычисляем (используя (I-П.2), (I-П.3) или (I-П.11)) собственные значения соответствующих картановских генераторов, ищем особые векторы и исследуем, какие из (под)модулей лежат в образе или в ядре экранирующих операторов. Действие экранируюших операторов на состояния из $\mathbb{G}_{r, p}$ определяется с помошью элементарных вычислений в конформной теории поля.

Середина диаграм.мbl. Начнем с состояния (2.9) и соответствующего $N=2$ модуля $\widetilde{\mathfrak{V}}_{\frac{1-r}{p}, p}(0,0, r-1)$. Вычисляя в этом модуле особый вектор $|E(r, 1, p)\rangle^{+}$(см. (I-П.4), (I-П.5)), видим, что он равен нулю. Из-за этого состояние $|1-r\rangle_{B C} \otimes|0, r-1\rangle_{\bar{A} A}$ удовлетворяет твистованному топологическому условию старшего веса

$$
|1-r\rangle_{B C} \otimes|0, r-1\rangle_{\bar{A} A} \doteq\left|\frac{r+1}{p}-1, p ;-r\right\rangle_{\text {top }} .
$$

Заметим теперь, что вектор (3.4) лежит в образе оператора $S_{B}$ :

$$
|1-r\rangle_{B C} \otimes|0, r-1\rangle_{\bar{A} A}=S_{B}|-r\rangle_{B C} \otimes|-1, r-1\rangle_{\bar{A} A},
$$


где мы имеем

$$
|-r\rangle_{B C} \otimes|-1, r-1\rangle_{\bar{A} A} \doteq\left|\frac{r+1}{p}-1,0, p ;-r\right\rangle .
$$

В модуле $\widetilde{\mathfrak{U}}_{\frac{r+1}{p}-1,0, p ;-r}$, порожденном из $|-r\rangle_{B C} \otimes|-1, r-1\rangle_{\bar{A} A}$, имеется заряженный особый вектор

$$
\begin{gathered}
\mathcal{G}_{-p} \ldots \mathcal{G}_{-r-1}|-r\rangle_{B C} \otimes|-1, r-1\rangle_{\bar{A} A}=|-p\rangle_{B C} \otimes|-1, r-1\rangle_{\bar{A} A}= \\
=S_{C}|1-p\rangle_{B C} \otimes|-1, r-p-1\rangle_{\bar{A} A}
\end{gathered}
$$

Возникаюший таким образом вектор $|1-p\rangle_{B C} \otimes|-1, r-p-1\rangle_{\bar{A} A}$ порождает модуль $\widetilde{\mathfrak{U}}_{\frac{r+1}{p}-2,0, p ; p-r}(p-r,-1, r-p-1)$, который и дает $(m=1, n=1)$ модуль из тех, что заполняют правое крыло. Заметим, что мы могли бы совершенно эквивалентным образом прийти к тому же модулю, сначала обратив внимание на тот факт, что $|0\rangle_{B C} \otimes|0, r-1\rangle_{\bar{A} A}=S_{C}|1\rangle_{B C} \otimes|0, r-p-1\rangle_{\bar{A} A}$, где, далее, $\mathcal{Q}_{r-p} \ldots \mathcal{Q}_{-1}|1\rangle_{B C} \otimes$ $|0, r-p-1\rangle_{\bar{A} A}=|p-r+1\rangle_{B C} \otimes|0, r-p-1\rangle_{\bar{A} A}=S_{B}|p-r\rangle_{B C} \otimes|-1, r-p-1\rangle_{\bar{A} A}$, причем $|p-r\rangle_{B C} \otimes|-1, r-p-1\rangle_{\bar{A} A}$ и $|1-p\rangle_{B C} \otimes|-1, r-p-1\rangle_{\bar{A} A}$ являются потомками друг друга. Центральная стрелка в (3.1) определяется, таким образом, как $S_{B} \circ S_{C}$ (что совпадает с $S_{C} \circ S_{B}$ с точностью до несушественного для нас знака).

Правое крыло бабочки. Припишем левому столбцу номер $n=1$, а верхней строке $m=1$. Тогда модуль в столбце $n$ и строке $m$, равный

$$
\begin{aligned}
& \widetilde{\mathfrak{U}}_{\frac{r+1}{p}-n-m, 0, p ; n p-r}(n p-r,-m, r-n p-1)= \\
& \quad=\widetilde{\mathfrak{U}}_{n+m-\frac{r+1}{p}, n+m-\frac{r+1}{p}, p ; 1-m p}(1-m p,-m, r-n p-1),
\end{aligned}
$$

порождается любым из векторов

$$
|n p-r\rangle_{B C} \otimes|-m, r-n p-1\rangle_{\bar{A} A} \doteq\left|\frac{r+1}{p}-m-n, 0, p ; n p-r\right\rangle
$$

или

$$
|1-m p\rangle_{B C} \otimes|-m, r-n p-1\rangle_{\bar{A} A} \doteq\left|n+m-\frac{r+1}{p}, n+m-\frac{r+1}{p}, p ; 1-m p\right\rangle .
$$

Заметим что равенство в (3.6) имеет место вследствие соотношений

$$
\begin{aligned}
& \mathcal{Q}_{-n p+r+1} \ldots \mathcal{Q}_{m p-1}|1-m p\rangle_{B C} \otimes|-m, r-n p-1\rangle_{\bar{A} A}= \\
& \quad=|n p-r\rangle_{B C} \otimes|-m, r-n p-1\rangle_{\bar{A} A}, \\
& |1-m p\rangle_{B C} \otimes|-m, r-n p-1\rangle_{\bar{A} A}= \\
& \quad=\mathcal{G}_{1-m p} \ldots \mathcal{G}_{n p-r-1}|n p-r\rangle_{B C} \otimes|-m, r-n p-1\rangle_{\bar{A} A}
\end{aligned}
$$

(которые выполняются с точностью до ненулевых множителей), и поэтому состояния $|1-m p\rangle_{B C} \otimes|-m, r-n p-1\rangle_{\bar{A} A}$ и $|n p-r\rangle_{B C} \otimes|-m, r-n p-1\rangle_{\bar{A} A}$ порождают один и тот же модуль. 


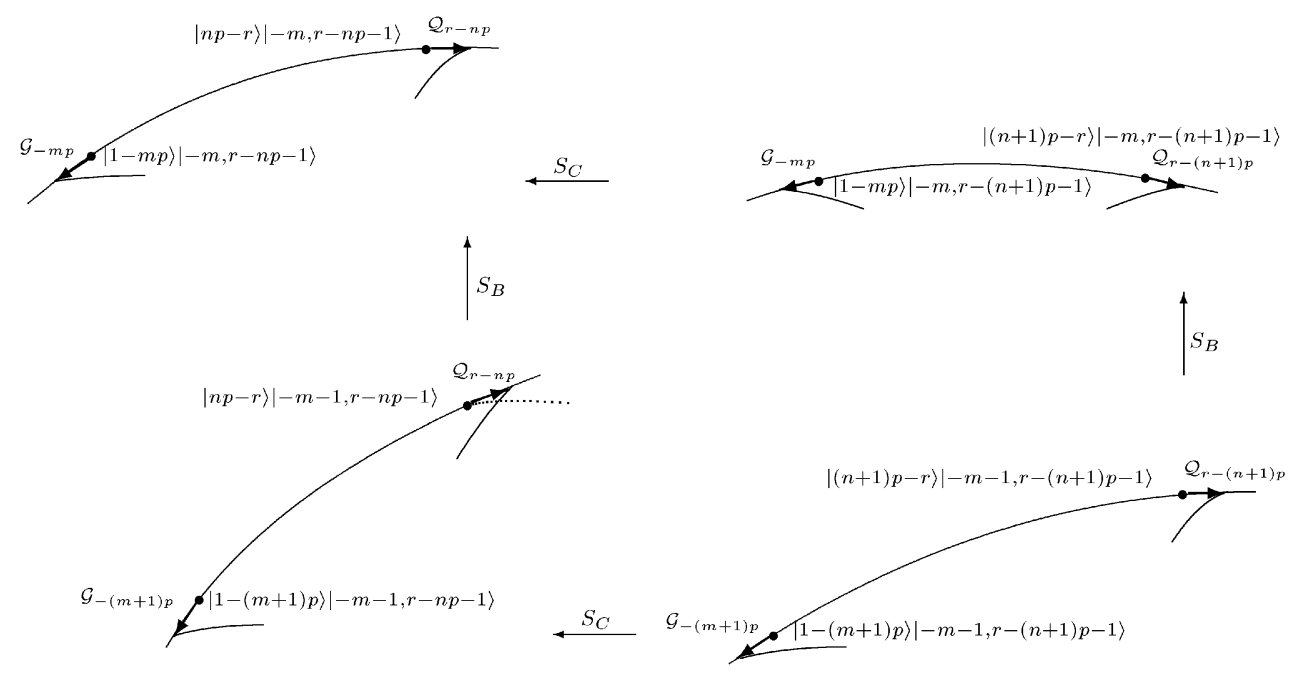

Экстремальные диаграммы модулей, отображаемых посредством экранирующих операторов.

Далее, прямые вычисления показывают, что векторы

$$
\mathcal{G}_{-m p}|1-m p\rangle_{B C} \otimes|-m, r-n p-1\rangle_{\bar{A} A} \doteq\left|n+m+\frac{1-r}{p}, p ;-m p\right\rangle_{\text {top }}
$$

и

$$
\mathcal{Q}_{r-n p}|n p-r\rangle_{B C} \otimes|-m, r-n p-1\rangle_{\bar{A} A} \doteq\left|\frac{r+1}{p}-n-m-1, p ; n p-r\right\rangle_{\text {top }}
$$

являются заряженными особыми векторами в модуле (3.6):

$$
\begin{array}{lc}
\mathcal{G}_{-m p}|1-m p\rangle_{B C} \otimes|-m, r-n p-1\rangle_{\bar{A} A} & \in \widetilde{\mathfrak{U}}_{\frac{r+1}{p}-m-n, 0, p ; n p-r} \ni \\
& \ni \mathcal{Q}_{r-n p}|n p-r\rangle_{B C} \otimes|-m, r-n p-1\rangle_{\bar{A} A} \\
\| & |1+n p-r\rangle_{B C} \otimes|-m, r-n p-1\rangle_{\bar{A} A} \\
|-m p\rangle_{B C} \otimes|-m, r-n p-1\rangle_{\bar{A} A} & \| \\
\| & S_{B}|n p-r\rangle_{B C} \otimes|-m-1, r-n p-1\rangle_{\bar{A} A} .
\end{array}
$$

Находя для векторов $|1-m p\rangle_{B C} \otimes|-m, r-(n+1) p-1\rangle_{\bar{A} A}$ и $|n p-r\rangle_{B C} \otimes \mid-m-1, r-$

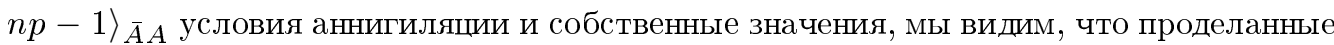
выше шаги применимы и к модулям, порожденным из этих векторов: каждый из них имеет два заряженных особых вектора, которые лежат в точности в образе одного из экранирующих операторов. 
"Перекрестные" комбинации двух экранирующих операторов получаются повторением тех же вычислений еще дважды. Это показано на рисунке, а результатом является сушествование отображений

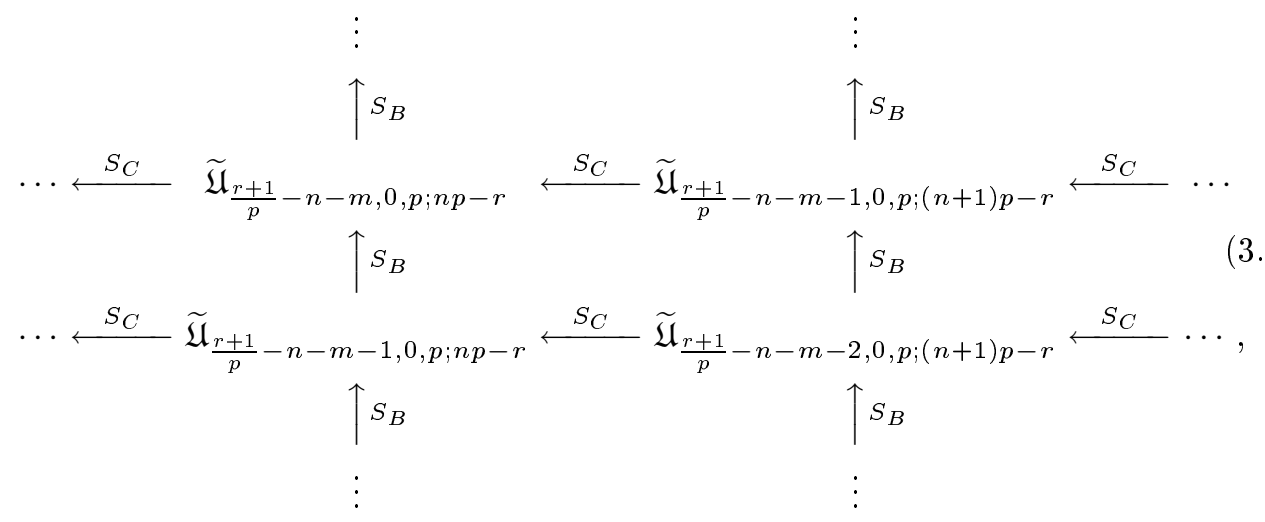

которые и составляют узор, заполняющий правое крыло бабочки. На рисунке для краткости опущены знаки тензорного умножения и индексы, обозначающие различные кетвекторы. Жирные точки изображают состояния, которые отображсаются в заряженные особые векторы под действием экранирующих операторов. Стрелки с надписями $\mathcal{Q}$ и $\mathcal{G}$ задают заряженные особые векторы. Пунктирная линия, проведенная в одном из случаев, показывает экстремальную диаграмму фактор-модуля по соответствующему особому вектору. Соответствующий подмодуль лежит в ядре экранирующего оператора, так что пунктирная линия отображается в экстремальную диаграмму подмодуля.

Левое крыло бабочки. Пронумеруем модули на левом крыле неотрицательными целыми $m$ и $n$ так, что $m=0$ соответствует нижней строке, а $n=0$ - вертикальной гранище. Модуль в положении $(m, n)$ получен склейкой модулей, порожденных из

$$
|-r-n p\rangle_{B C} \otimes|m, n p+r-1\rangle_{\bar{A} A} \doteq\left|\frac{r+1}{p}+m+n, 0, p ;-r-n p\right\rangle
$$

$$
\begin{aligned}
|m p+1\rangle_{B C} \otimes|m, n p+r-1\rangle_{\bar{A} A} \doteq \\
\quad \doteq\left|-\frac{r+1}{p}-m-n,-\frac{r+1}{p}-m-n, p ; m p+1\right\rangle .
\end{aligned}
$$

Склейка основана на том факте, что в модуле, порожденном из (3.8), имеется подмодуль, порожденный из особого вектора

$$
\begin{aligned}
\mathcal{Q}_{r+n p} \mid & -r-n p\rangle_{B C} \otimes|m, n p+r-1\rangle_{\bar{A} A}=|1-r-n p\rangle_{B C} \otimes|m, n p+r-1\rangle_{\bar{A} A} \doteq \\
& \doteq\left|\frac{r+1}{p}+m+n-1, p ;-r-n p\right\rangle_{\text {top }},
\end{aligned}
$$


и что mom жее подмодуль порождается также из особого вектора

$$
\mathcal{G}_{m p}|m p+1\rangle_{B C} \otimes|m, n p+r-1\rangle_{\bar{A} A}=|m p\rangle_{B C} \otimes|m, n p+r-1\rangle_{\bar{A} A}
$$

в модуле, порожденном из (3.9), поскольку состояния $|1-r-n p\rangle_{B C} \otimes|m, n p+r-1\rangle_{\bar{A} A}$ и $|m p\rangle_{B C} \otimes|m, n p+r-1\rangle_{\bar{A} A}$ являются потомками друг друга.

Применяя экранирующие операторы, получаем

$$
\begin{gathered}
S_{B}|-r-n p\rangle_{B C} \otimes|m, n p+r-1\rangle_{\bar{A} A}=|1-r-n p\rangle_{B C} \otimes|m+1, n p+r-1\rangle_{\bar{A} A}= \\
\quad=\mathcal{Q}_{r+n p}|-r-n p\rangle_{B C} \otimes|m+1, n p+r-1\rangle_{\bar{A} A}
\end{gathered}
$$

и

$$
\begin{gathered}
S_{C}|m p+1\rangle_{B C} \otimes|m, n p+r-1\rangle_{\bar{A} A}=|m p\rangle_{B C} \otimes|m,(n+1) p+r+1\rangle_{\bar{A} A}= \\
=\mathcal{G}_{m p}|1+m p\rangle_{B C} \otimes|m,(n+1) p+r+1\rangle_{\bar{A} A}
\end{gathered}
$$

откуда видно, что узор (3.8)-(3.11) воспроизводится в (3.12) и (3.13) с соответствующими заменами $m \mapsto m+1$ и $n \mapsto n+1$. В модуле, порожденном из (3.8) и (3.9), $\operatorname{Ker} S_{B}$ порождается из (3.9), a $\operatorname{Ker} S_{C}$ - из (3.8), причем $\operatorname{Ker} S_{B} \cap \operatorname{Ker} S_{C}$ - подмодуль, порожденный из (3.10). Таким образом заполняется все левое крыло (узор которого оказывается в очевидном смысле двойственным узору правого крыла).

Напомним теперь стандартный факт, состоящий в том, что всякая горизонтальная или вертикальная последовательность отображений, осушествляемых одним фермионным экранирующим оператором, представленным в виде вершинного оператора, точна (кроме, очевидно, границы крыла, если последовательность продолжается посредством $\cdots \rightarrow 0$ или $0 \rightarrow \cdots)$. Таким образом, отображения точны везде, кроме угла. Центральное отображение дается произведением двух экранируюших операторов. Когомологии определяются исходя из того, что $\operatorname{Im} S_{B}=\operatorname{Im} S_{C}=\widetilde{\mathfrak{V}}_{\frac{1-r}{p}, p}(0,0, r-1)$, a $\operatorname{Im} S_{B} \circ S_{C}$ - максимальный подмодуль в $\widetilde{\mathfrak{V}}_{\frac{1-r}{p}, p}(0,0, r-1)$. Более экономный способ, приводящий к тому же результату, состоит в том, чтобы отобразить резольвенту-бабочку в двустороннюю резольвенту (I-3.18), как это описано в следуюшем разделе.

\section{4. СМИНАНИЕ БАБОЧКИ В ДВУСТОРОННЮЮ РЕЗОЛЬВЕНТУ}

Как известно [10], ядро или коядро фермионного экранирующего оператора порождает $\beta \gamma$-систему. Мы используем это применительно к резольвенте-бабочке, в результате чего возникнет в точности $\beta \gamma$-система из раздела I-3 (а резольвента-бабочка превратится в “линейную” двустороннюю резольвенту (I-3.18)). В самом деле, определим фермионную систему первого порядка

$$
\eta=C e^{p \bar{X}}, \quad \xi=B e^{-p \bar{X}},
$$


а также введем два скаляра $\phi$ и $\varphi$ с сигнатурами -1 и +1 :

$$
\partial \phi=B C+\frac{1}{p} A-p \bar{A}, \quad \partial \varphi=B C+\frac{1}{p} A .
$$

Второй скаляр представляет фермионную $b c$-систему из (I-4.16). Взяв теперь $\eta, \xi$ и $\phi$ из (4.1), (4.2), мы введем бозонную систему первого порядка в соответствии с (I-4.20). Тогда $N=2$ генераторы (2.1) перепишутся через эти $(b, c, \beta, \gamma)$-поля как

$$
\mathcal{Q}=-\frac{1}{p} \beta c, \quad \mathcal{G}=(p-1) b \partial \gamma-\gamma \partial b,
$$

что отличается от соответствующих генераторов в (I-3.4) только заменами $\mathcal{Q} \mapsto p \mathcal{Q}$, $\mathcal{G} \mapsto \frac{1}{p} \mathcal{G}$, не меняющими коммутационных соотношений (а вся остальная $N=2$ алгебра порождается операторами $\mathcal{Q}$ и $\mathcal{G}$ ). Экранируюший оператор $S_{C}$ становится теперь просто оператором $\eta_{0}$, который “редуцирует” $\eta \xi \phi$-модуль к $\beta \gamma$-модулю путем устранения $\xi_{0}$-моды. Второй фермионный экранируюший оператор при этом принимает вид

$$
S_{B}=\frac{1}{2 \pi i} \oint c \gamma^{p-1}
$$

что совпадает с оператором $\mathbb{Q}_{0}$ из раздела I-3.

Резольвенту-бабочку можно отобразить на двустороннюю резольвенту (I-3.18) путем сминания ее крыльев таким образом, что остаются только $S_{B}$-отображения. Рассмотрим сначала правое крыло. В нем сохраним только вертикальную гранищу (см. (3.1)) и возьмем фактор по образу $S_{C}$ в каждом модуле. В результате остается только $b c \beta \gamma$-модуль (т.е. когомологии последовательности $0 \stackrel{S_{C}}{\longleftarrow} \cdots \stackrel{S_{C}}{\longleftarrow} \cdots$ ). Полагая в (3.6) $n=1$, мы получаем отображения

$$
\widetilde{\mathfrak{U}}_{\frac{r+1}{p}-m-1,0, p ; p-r}(p-r,-m, r-p-1) \stackrel{S_{B}}{\longrightarrow} \widetilde{\mathfrak{U}}_{\frac{r+1}{p}-m, 0, p ; p-r}(p-r,-m+1, r-p-1) .
$$

С другой стороны, образ оператора $S_{C}$ является подмодулем в $\widetilde{\mathfrak{U}}_{\frac{r+1}{p}-m-1,0, p ; p-r}(p-$ $r,-m, r-p-1)$, порожденным из вектора

$$
|-m p\rangle_{B C} \otimes|-m, r-p-1\rangle_{\bar{A} A} \doteq\left|\frac{1-r}{p}+m, p ;-p\right\rangle_{\text {top }} .
$$

При этом фактор-модуль порожден из вектора

$$
|1-m p\rangle_{B C} \otimes|-m, r-p-1\rangle_{\bar{A} A} \doteq\left|\frac{1-r}{p}+m, p ;-m p\right\rangle_{\text {top }},
$$

где $\doteq$ выполнено в факторе, т.е. по модулю вектора (4.5). Таким образом, отображение (4.4) индуцирует последовательность

$$
\begin{aligned}
\ldots & \stackrel{S_{B}}{\longrightarrow} \widehat{\mathfrak{V}}_{\frac{1-r}{p}+m+1, p ;-(m+1) p}(1-(m+1) p,-m-1, r-p-1) \stackrel{S_{B}}{\longrightarrow} \\
& \stackrel{S_{B}}{\longrightarrow} \widehat{\mathfrak{V}}_{\frac{1-r}{p}+m, p ;-m p}(1-m p,-m, r-p-1) \stackrel{S_{B}}{\longrightarrow} \cdots \stackrel{S_{B}}{\longrightarrow} \\
& \stackrel{S_{B}}{\longrightarrow} \widehat{\mathfrak{V}}_{\frac{1-r}{p}+1, p ; 0}(1-p,-1, r-p-1),
\end{aligned}
$$


где $\widehat{\mathfrak{V}}$ - модули, в которых профакторизовано по особому вектору $\mathcal{G}_{-m p}|1-m p\rangle_{\text {ВC }} \otimes$ $|-m, r-p-1\rangle_{\bar{A} A}$ (см. левый верхний угол экстремальной диаграммы на рисунке).

На левом крыле возьмем модули на вертикальной границе $(n=0$ в $(3.8),(3.9))$ и оставим в них только ядра оператора $S_{C}$ (т.е. когомологии последовательности $\left.0 \stackrel{S_{C}}{\longrightarrow} \cdots \stackrel{S_{C}}{\longrightarrow} \cdots\right)$. Ядро $S_{C}$ порождается из вектора

$$
|-r\rangle_{B C} \otimes|m, r-1\rangle_{\bar{A} A} \doteq\left|\frac{r+1}{p}+m, 0, p ;-r\right\rangle, \quad m \geqslant 0
$$

Обозначая этот модуль через $\widehat{\mathfrak{U}}_{m+\frac{r+1}{p}, 0, p ;-r}(-r, m, r-1)$, получаем

$$
\begin{aligned}
\widehat{\mathfrak{U}}_{\frac{r+1}{p}, 0, p ;-r}(-r, 0, r-1) & \stackrel{S_{B}}{\longrightarrow} \widehat{\mathfrak{U}}_{\frac{r+1}{p}+1,0, p ;-r}(-r, 1, r-1) \stackrel{S_{B}}{\longrightarrow} \\
& \stackrel{S_{B}}{\longrightarrow} \widehat{\mathfrak{U}}_{\frac{r+1}{p}+2,0, p ;-r}(-r, 2, r-1) \stackrel{S_{B}}{\longrightarrow} \cdots,
\end{aligned}
$$

что позволяет продолжить последовательность (4.7). Тем самым воспроизводится комплекс (I-3.18), подвергнутьй спектральному потоку на $\theta=1-r$.

Возврашаясь к резольвенте-бабочке, мы видим, что ее когомологии являются унитарным $N=2$ представлением.

ЗАМЕчАНИЕ 4.1. Заметим, что бозонный экранирующий оператор реализации (2.1)

$$
S_{\mathrm{W}}=\frac{1}{2 \pi i} \oint(p \bar{A}-B C) e^{-\frac{1}{p} X-\bar{X}}
$$

теперь принимает вид (I-4.21). Этот оператор не участвует в построении резольвент в данной статье.

ЗАмЕЧАНИЕ 4.2. После отображения в представление Вакимото экранируюший оператор

$$
S_{C}=\frac{1}{2 \pi i} \oint \eta
$$

как и раньше, служит для корректного определения пространства представления бозонной системы первого порядка, на этот раз $\boldsymbol{\beta} \boldsymbol{\gamma}$-системы из [1] (таким образом, этот оператор становится ненужным, коль скоро построение проводится в терминах самих полей $\boldsymbol{\beta}$ и $\boldsymbol{\gamma}$, а не их бозонизации). С другой стороны, фермионный экранирующий оператор $S_{B}$ принимает вид (I-4.23) (отметим здесь восстановление, наконец-то, "стандартной" вертексной формы этого оператора).

\section{5. ЗАКЛЮЧЕНИЕ}

Сравнивая двустороннюю “линейную” резольвенту для унитарных $N=2$ представлений из статьи [1] и бабочку, мы видим, конечно, “сильную зависимость” вида резольвенты от выбранных свободных полей. В действительности форма резольвенты в значительной степени определяется квантовой группой, закодированной в структуре экранируюших операторов. 
Что касается резольвенты-бабочки, ее форму можно полуэвристически объяснить как эффект “фельдеровского типа," происходящий в “ $3,5,7, \ldots$ ”-резольвенте неприводимых $N=2$ представлений [9]. Эти резольвенты строятся из твистованных массивных $x$ модулей Верма (см. определение I-П.2) и выглядят как

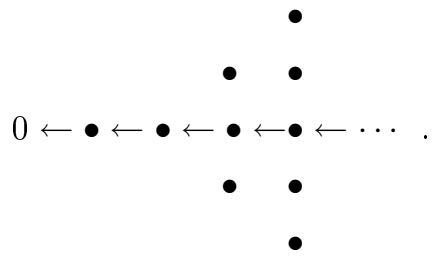

Складывая (несколько несимметричным образом) крылья бабочки в (1.1), получаем как раз структуру (5.1). Можно говорить поэтому, что $b c \beta \gamma$-реализация $N=2$ модулей “выворачивает наизнанку" некоторые из отображений в (5.1), что и дает (1.1). Резольвенту Фельдера тоже можно рассматривать подобным образом, когда "косичка" вида (I-П.9) становится бесконечной линией, а когомологии концентрируются в середине. Этому можно придать и точный смысл [4]. Интересным вопросом в связи с этим является построение "промежуточных" модулей, интерполируюших между “ $3,5,7, \ldots$. '-резольвентой и бабочкой.

Аналогичная резольвента-бабочка для унитарных представлений алгебры $\widehat{s \ell}(2)$ состоит из твистованных (подвергнутых спектральному потоку) модулей Вакимото. Картины [10] $\boldsymbol{\beta} \boldsymbol{\gamma}$-системы из [1] оказываются различными для различных модулей, так что эту резольвенту можно рассматривать как пример резольвенты, относяшейся к трехбозонной реализации, получаемой дополнительной бозонизацией входящих в представление Вакимото $\beta \boldsymbol{\gamma}$-полей.

Заметим, что унитарные $N=2$ представления характеризуются соотношением

$$
\partial^{p-2} \mathcal{G}(z) \ldots \partial \mathcal{G}(z) \mathcal{G}(z)=0
$$

Это дает альтернативный способ показать, что когомологии являются суммой унитарных представлений: достаточно проверить действие $N=2$ алгебры и убедиться, что формула (5.2) удовлетворяется (в $b c \beta \gamma$-реализации, например, это условие выполнено, поскольку поле $\gamma(z)$ удовлетворяет в когомологиях соотношению $\left.\gamma^{p-1}=0\right)$. С точки зрения моделей Гинзбурга-Ландау соотношение (5.2) обнаруживает формальную связь с невозмушенными $A_{p-1}$-уравнениями движения $X^{p-1}=0$ (см. (I-3.2)). Можно развить эту аналогию, вспомнив, что киральное кольцо в теории Гинзбурга-Ландау порождено элементами $1, X, \ldots, X^{p-2} ;$ унитарное же представление $N=2$ алгебры можно породить действием только мод $\mathcal{G}_{n}, n \in \mathbb{Z}$, подчиненных связи, следующей из (5.2), без участия каких бы то ни было других $N=2$ генераторов [11].

Благодарности. Эта работа частично поддержана грантом РФФИ № 98-01-01155 и грантом № 99-15-96037 Президента Российской Федерации. 


\section{Список литературы}

[1] А. М. Семихатов. ТМФ. 1999. Т. 121. № 1. С. 89

[2] G. Felder. Nucl. Phys. B. 1989. V. 317. P. 215; E: B. 1989. V. 324 . P. 548.

[3] D. Bernard, G. Felder. Commun. Math. Phys. 1990. V. 127. P. 145.

[4] B. L. Feigin, E. V. Frenkel. Representations of Affine Kac-Moody Algebras and Bosonization. In: Physics and Mathematics of Strings. Memorial Volume for Vadim Knizhnik. Eds. L. Brink, D. Friedan, A. M. Polyakov. Singapore: World Sci., 1993; B. L. Feigin, E. V. Frenkel. Commun. Math. Phys. 1990. V. 128. P. 161.

[5] P. Bouwknegt, J. McCarthy, K. Pilch. Commun. Math. Phys. 1990. V. 131. P. 125.

[6] G. Mussardo, G. Sotkov, M. Stanishkov. Int. J. Mod. Phys. A. 1989. V. 4. P. 1135.

[7] N. Ohta, H. Suzuki. Nucl. Phys. B. 1990. V. 332. P. 146.

[8] K. Ito. Phys. Lett. B. 1991. V. 259. P. 73; Nucl. Phys. B. 1992. V. 370. P. 123.

[9] B. L. Feigin, A. M. Semikhatov, V. A. Sirota, I. Yu. Tipunin. Nucl. Phys. B. 1999. V. 536. P. 617 ; hep-th $/ 9805179$.

[10] D. H. Friedan, E. J. Martinec, S. H. Shenker. Nucl. Phys. B. 1986. V. 271. P. 93.

[11] B. L. Feigin, A. M. Semikhatov, I. Yu. Tipunin. A Semi-Infinite Realization of Unitary Representations of the $N=2$ Superconformal Algebra (в работе).

Поступила в редакцию 14. V.1999 г. 